Case Report

\title{
Accumulation of brown pigment-laden macrophages associated with vascular lesions in the lungs of cynomolgus monkeys (Macaca fascicularis)
}

\author{
Yoshika Yamakawa $^{1 *}$, Tetsuya Ide ${ }^{1}$, Hikaru Mitori $^{1}$, Yuji Oishi ${ }^{1}$, and Masahiro Matsumoto ${ }^{1}$ \\ ${ }^{1}$ Drug Safety Research Labs., Astellas Pharma Inc., 21 Miyukigaoka, Tsukuba, Ibaraki 305-8585, Japan
}

\begin{abstract}
Accumulation of macrophages containing brown pigments in the lungs is a well-known spontaneous lesion found in cynomolgus monkey. However, its pathogenesis has not been clearly described. In our survey, brown pigment-laden macrophages were found in the lungs of 4 out of 43 cases. Brown pigments were mostly found in the macrophages of the perivascular interstitium, which proved to be hemosiderin. Some small- to medium-sized vessels that exhibited prominent accumulation of brown pigment-laden macrophages showed degeneration and necrosis of the smooth muscle cells of tunica media. Furthermore, ruptures of the internal and external elastic laminae were seen in some of the vessels. These findings suggested that partial fragmentation of the vascular elastic lamina followed by degeneration and necrosis of the tunica media caused blood leakage leading to the accumulation of hemosiderinladen macrophages in the perivascular interstitium of the lungs. (DOI: 10.1293/tox.2015-0079; J Toxicol Pathol 2016; 29: 181-184)
\end{abstract}

Keywords: brown pigmentation, hemosiderin, vascular lesions, lung, cynomolgus monkey

Brown pigmentation in the lungs of cynomolgus monkeys is a well-known spontaneous pathological finding ${ }^{1-3}$. However, the origin of the brown pigment as well as the underlying mechanism has not been clearly described so far. In order to obtain some clues to the pathogenesis of these lesions, here we report the features and the distribution of brown pigmentation along with associated vascular lesions in cynomolgus monkeys.

Lung tissue samples from a total of 43 cynomolgus monkeys ( 22 males, 21 females) were collected from preclinical toxicity studies that included both test article-treated and non-treated animals and were conducted in the Kashima Facilities of Astellas Pharma Inc., between 2012 and 2013. All animals were purchased from Japan Laboratory Animals, Inc. (Tokyo, Japan), and had been bred in Cambodia. For all animals, serological examination for viral diseases (Cercopithecine herpesvirus 1 (B virus), simian immunodeficiency virus (SIV), simian T-cell leukemia virus (STLV), simian retroviruses (SRV), and Ebola), bacteriological examination (Mycobacterium tuberculosis, Shigella spp, and Salmonella spp), and microscopic or fecal smear examination for parasites (lice, fleas, ticks, Giardia, Strongyloides, Entamoeba)

Received: 11 December 2015, Accepted: 1 March 2016

Published online in J-STAGE: 25 March 2016

*Corresponding author: Y Yamakawa

(e-mail: yoshika.yamakawa@astellas.com)

(C)2016 The Japanese Society of Toxicologic Pathology

This is an open-access article distributed under the terms of the Creative Commons Attribution Non-Commercial No Derivatives (by-ncnd) License $<$ http://creativecommons.org/licenses/by-nc-nd/4.0/> . had been carried out and had confirmed that the animals were free of the above-mentioned agents prior to purchase. They were individually housed in stainless steel cages $(630$ $\mathrm{mm} \mathrm{W} \times 650 \mathrm{~mm} \mathrm{D} \times 1180 \mathrm{~mm} \mathrm{H})$ in a room maintained at a temperature and humidity of $24 \pm 1{ }^{\circ} \mathrm{C}$ and $58 \pm 12 \%$, respectively, with an average of 17.3 air ventilation changes/ hour (range: 13.8-20.8 changes/hour) and an artificial light cycle of 12 hours/day (7:00 to 19:00). The animals were fed with commercial primate diet (PS; Oriental Yeast Co., Ltd., Tokyo, Japan) and allowed ad libitum access to water. All animal experimental procedures were approved by the Institutional Animal Care and Use Committee of Astellas Pharma Inc. Furthermore, the Kashima Facilities of Astellas Pharma Inc., were awarded Accreditation Status by the AAALAC International.

Each animal was anesthetized with an intramuscular injection of ketamine $(20 \mathrm{mg} / \mathrm{kg})$ and euthanized by exsanguination at the end of the study period, and a full internal and external macroscopic examination was performed.

Lungs were fixed in $10 \%$ neutral buffered formalin and the right caudal lobe and left cranial lobe were processed for routine paraffin embedding. The paraffin sections were stained with hematoxylin and eosin (HE). Lung sections which showed brown pigment-laden macrophages were evaluated based on the percentage of the vessels associated with brown pigment-laden macrophages using the following criteria: mild, less than $25 \%$; moderate, $25-50 \%$; and severe, more than $50 \%$ of the vessels associated with pigmentation. Four cases which showed brown pigmentation histopathologically, were further analyzed by Berlin Blue and Victoria Blue staining, immunohistochemistry for anti- 
bodies against Ionized calcium binding adapter molecule 1 (Iba-1;Wako Pure Chemical Industries, Ltd., Osaka, Japan) and Podoplanin (D2-40, M3619, monoclonal mouse anti-human, Dako, Tokyo, Japan), and one severe case (case 1) was further examined by electron microscopy. For electron microscopic examination, formalin-fixed lung tissue was further fixed in $1 \%$ osmium, routinely processed and embedded in Epon 812. After semi-thin sections stained with Toluidine Blue were examined by light microscopy, ultrathin sections were cut from selected blocks, stained with uranyl acetate and lead citrate and examined under a transmission electron microscope (H-7600, Hitachi, Tokyo, Japan).

As a result, brown pigment-laden macrophages in the lungs were observed in 4 out of 43 animals (9\%), ranging from mild to marked severity (Table 1). The affected animals were all males and approximately 5 years of age at the time of necropsy.

Cases 1 and 3 had no noteworthy findings in clinical observations, body weight, food consumption, hematology, and blood chemistry throughout the quarantine, acclimation, and toxicity study periods. Cases 2 and 4 had various clinical signs related to dosing of the test article during the treatment period; however, as no changes were seen in the blood coagulation system, blood pressure, or heart rate, it was concluded that they were not related to brown pigmentation of the lungs.

At necropsy, all lung lobes of case 1 which suffered severe accumulation of brown pigment-laden macrophages, showed a brown appearance, but the other three cases had no gross findings in the lungs.

Histologically, whereas brown pigment-laden macrophages were observed around small- to medium-sized vessels only within interlobular areas in mild cases (cases 3 and 4), in moderate and severe cases (cases 2 and 1, respectively), these brown pigment-laden macrophages accumulated not only around small- to medium-sized vessels within interlobular areas, but also diffusely distributed in subpleural areas of the lung (Fig. 1a, c). Moreover, in the severe case (case 1), hemorrhage was seen around these vessels. In all four cases, pigment-laden macrophages were hardly seen within intraalveolar spaces. Vessels, regardless of sizes, that had a well-defined lumen, rounded shape and were maintained by a thick tunica media and abundant elastic fibers were judged as arteries and vessels that were irregularly shaped with thin walls and no or poorly developed elastic fibers were assumed to be veins which were discriminated using Victoria Blue-HE double stained sections. There was no difference in amount of macrophage accumulation between arteries and veins. Besides, there was no brown pigment deposition in perivascular areas in other organs in any of the cases.

Immunohistochemical staining for Iba-1 confirmed that brown pigments were phagocytosed by macrophages (Fig. 1d). In order to identify the detailed distribution of brown pigment-laden macrophages, immunohistochemical staining for Podoplanin, a specific marker for lymphatic endothelium, was performed. As a result, brown pigmentladen macrophages were not found within lymphatic ducts (Fig. 1e). These macrophages were only distributed in the interstitium surrounding vessels.

Brown pigments observed in the macrophage were positive for Berlin Blue staining, indicative of hemosiderin (Fig. 1b). Furthermore, electron microscopic examination showed that brown pigments were presumably intracellular siderosomes containing electron-dense fine granules (i.e. ferritin aggregates) (Fig. 2).

The smooth muscle cells of the tunica media of smallto medium-sized vessels with prominent perivascular pigmentation showed degeneration and necrosis focally. Moreover, these vessels partially lacked an elastic lamia which was confirmed by Victoria Blue-HE double staining (Fig. 3a, b). The external elastic lamina tended to be more affected than the internal elastic lamina, and a larger number of brown pigment-laden macrophages was observed where fragmentation was present. On the other hand, areas with mild fragmentation of the elastic lamina showed fewer numbers of hemosiderin-laden macrophages. However, neither degeneration/necrosis of smooth muscle cells of the tu-

Table 1. Summary of Histopathological Findings in Cynomolgus Monkeys with Pigment-laden Macrophages

\begin{tabular}{|c|c|c|c|c|c|}
\hline & $\begin{array}{c}\text { Case No. } \\
\text { Age } \\
\text { Sex }\end{array}$ & $\begin{array}{c}\text { Case } 1 \\
5 \text { Y } 2 \text { M } \\
\text { Male }\end{array}$ & $\begin{array}{c}\text { Case } 2 \\
5 \text { Y } 1 \mathrm{M} \\
\text { Male }\end{array}$ & $\begin{array}{c}\text { Case } 3 \\
5 \text { Y } 3 \text { M } \\
\text { Male }\end{array}$ & $\begin{array}{c}\text { Case } 4 \\
5 \text { Y } 5 \text { M } \\
\text { Male }\end{array}$ \\
\hline \multicolumn{6}{|l|}{ Localization/histological findings } \\
\hline \multicolumn{6}{|l|}{ Small- to medium-sized bronchial arteries/veins } \\
\hline Accumulation of brown pigment-laden macrophages & & +++ & ++ & + & + \\
\hline Hemorrhage & & + & + & - & - \\
\hline Fragmentation of elastic lamina & & ++ & + & + & + \\
\hline Degeneration/necrosis of tunica media & & ++ & + & - & - \\
\hline \multicolumn{6}{|l|}{ Large pulmonary arteries/veins } \\
\hline Accumulation of brown pigment-laden macrophages & & + & - & - & - \\
\hline Hemorrhage & & - & - & - & - \\
\hline Fragmentation of elastic lamina & & - & - & - & - \\
\hline Degeneration/necrosis of tunica media & & - & - & - & - \\
\hline Vessels in other organs & & - & - & - & - \\
\hline
\end{tabular}

Y, years; M, months, Grade: -, no change; +, mild; ++, moderate; +++, severe. 

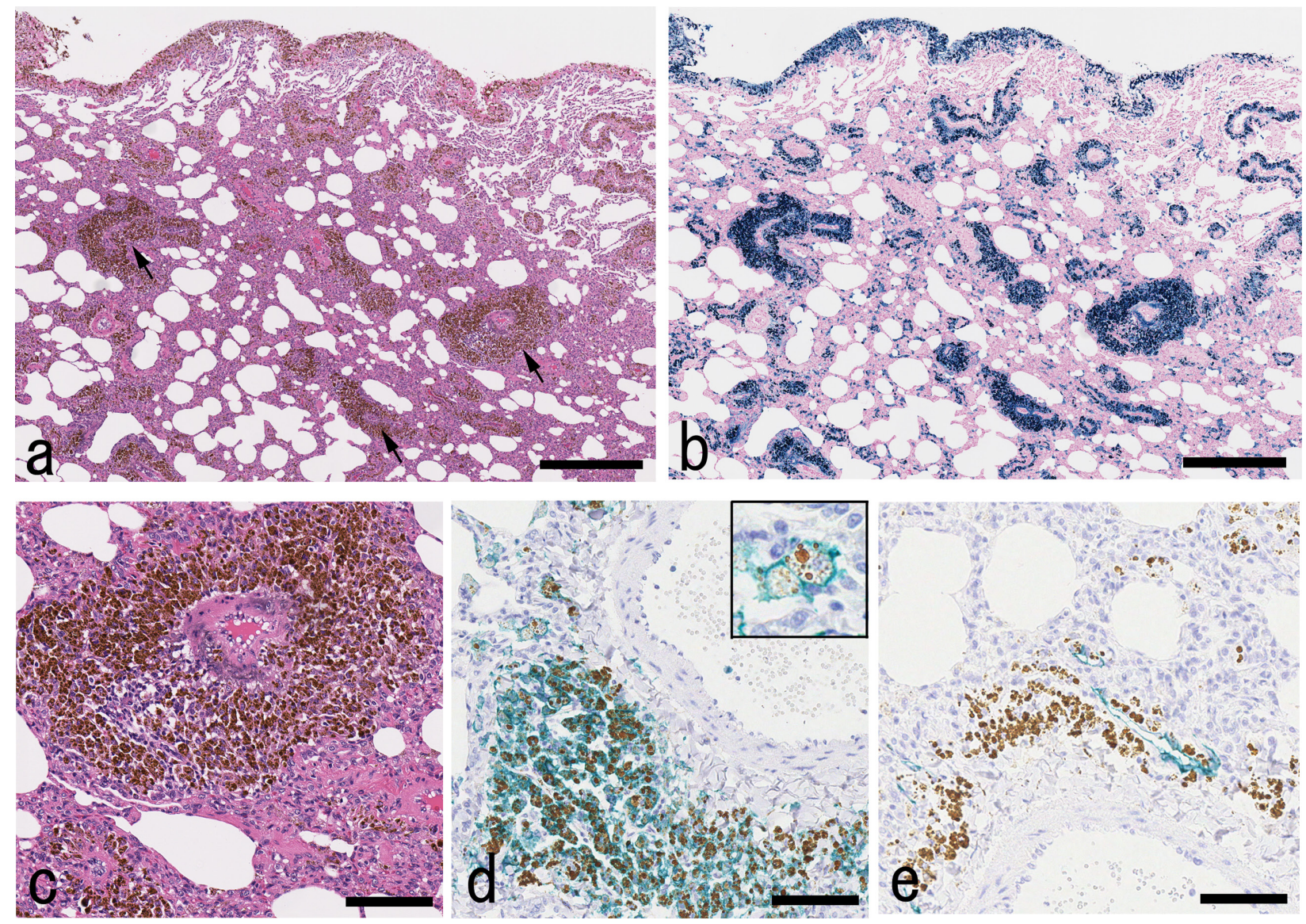

Fig. 1. Histopathological findings in the lung of a cynomolgus monkey (case 1). a) Multifocally, large numbers of brown pigment-laden macrophages were observed mainly around bronchial arteries (arrows). HE. Bar $=500 \mu \mathrm{m}$. b) Brown pigments were positive for Berlin Blue staining, suggestive of hemosiderin. Bar $=500 \mu \mathrm{m}$. c) Brown pigment deposition was prominent around small- to medium-sized bronchial arteries within interlobular areas. HE. Bar $=100 \mu \mathrm{m}$. d) Immunohistochemical staining for Iba-1 showed that brown pigments were phagocytosed by Iba-1-positive macrophages (inset). Bar $=100 \mu \mathrm{m}$. e) Immunohistochemical staining for Podoplanin, a specific marker for lymphatic endothelium revealed that brown pigments were not found within perivascular lymphatic ducts. Bar $=80 \mu \mathrm{m}$.

nica media nor fragmentation of the elastic lamina was seen in larger vessels such as pulmonary arteries and veins.

It has been reported by Sato et al. that accumulation of brown pigment-laden macrophages around pulmonary vessels is a rare spontaneous lesion based on a survey of 660 cynomolgus monkeys used in toxicity studies performed between $1998-2011^{2}$. In the current survey, $9 \%$ of cynomolgus monkeys had hemosiderin deposition surrounding most likely bronchial arteries and veins of their lungs which was considered to be a higher incidence compared with that in the previous report by Sato et al. ${ }^{2}$

Rupture of the vascular elastic lamina was seen in all cases; however, degeneration and necrosis of smooth muscle cells of the tunica media were found in severe and moderate cases (cases 1 and 2, respectively) but not in mild cases (Table 1). Taken together, it was speculated that partial fragmentation of the pulmonary vascular elastic lamina extended to degeneration and necrosis in smooth muscle of the tunica media which caused blood leakage to the surrounding interstitium, consequently leading to hemosiderin deposition. Although it is not known whether these cyno-

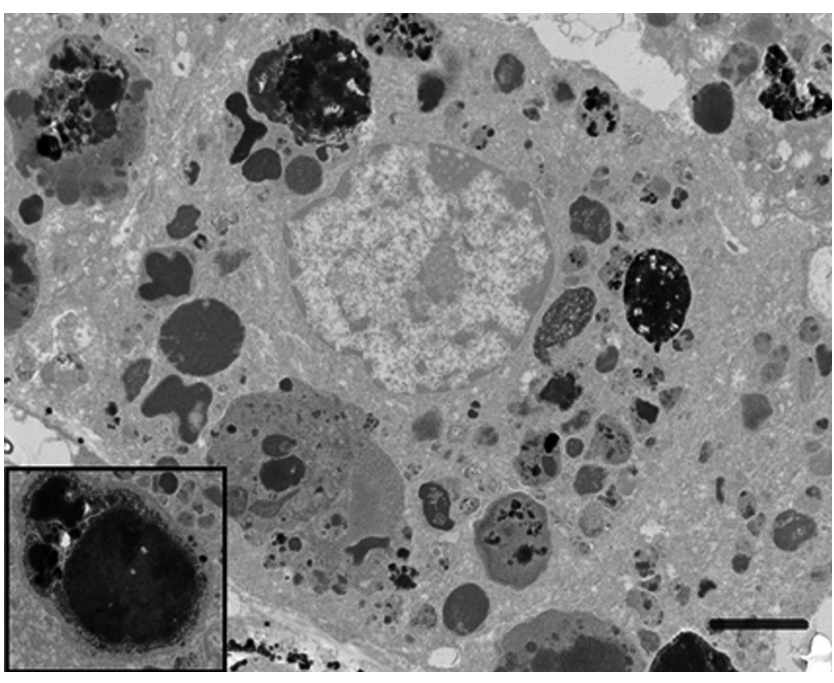

Fig. 2. Electron micrograph of pigment-laden macrophages in the lung of a cynomolgus monkey (case 1). Brown pigments were assumed to be intracellular siderosomes containing electrondense fine granules (inset). $\mathrm{Bar}=2 \mu \mathrm{m}$. 


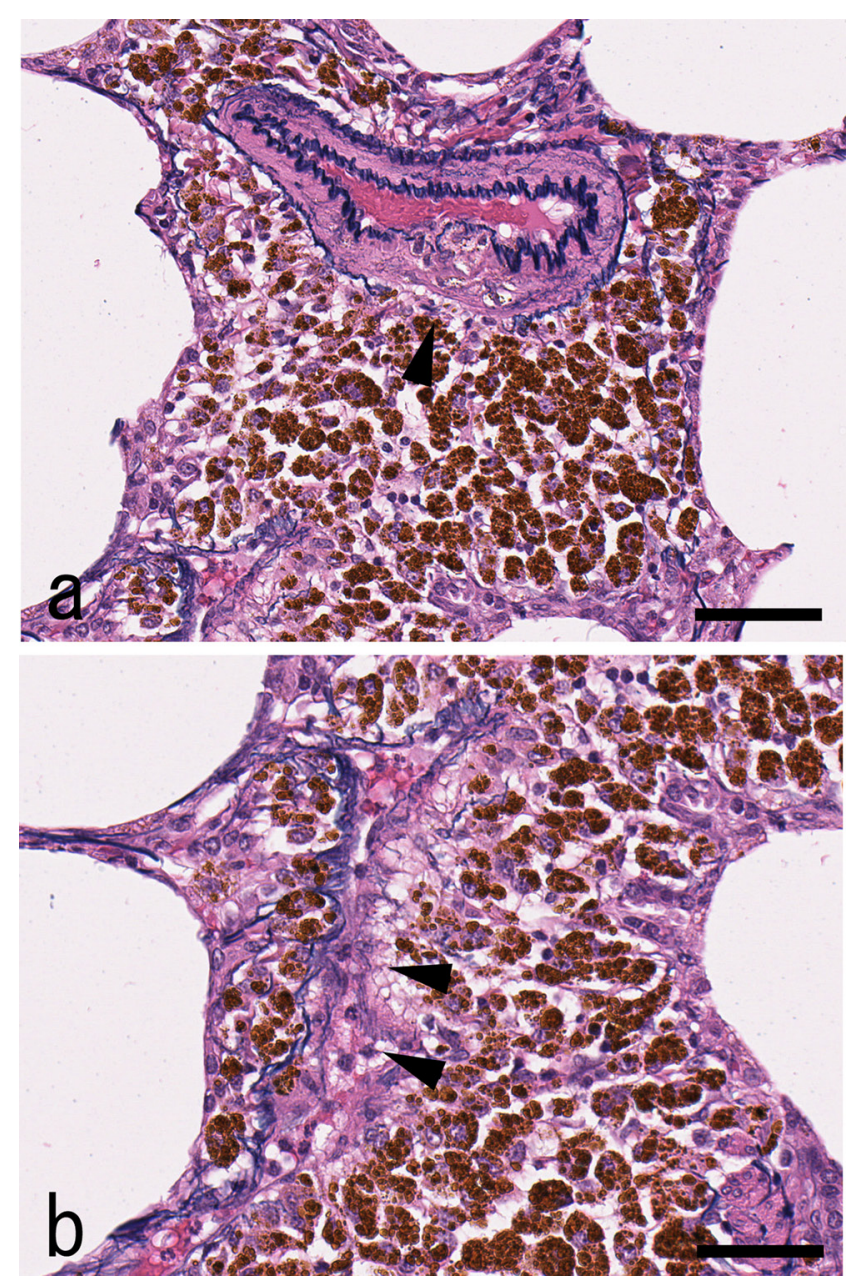

Fig. 3. Victoria Blue-HE double staining of pulmonary vessels in a cynomolgus monkey (case 1). a) The smooth muscle cells of the tunica media of an artery showed degeneration and necrosis on top of partial loss of the elastic lamina (arrowhead) where large numbers of hemosiderin-laden macrophages were accumulated. b) Partial loss of the elastic lamina was also seen in a vein (arrowheads) where large numbers of hemosiderin-laden macrophages were present. $\mathrm{Bar}=60 \mu \mathrm{m}$.

molgus monkeys were genetically related, they were all bred in Cambodia and purchased at a relatively young age; it can be assumed that the present findings are congenital lesions possibly due to abnormal function or structure of vessels.

The lung is functionally divided into pulmonary and bronchial circulation. The pulmonary circulation (i.e. functional vessel) nourishes the alveolar parenchyma and distal bronchioles and alveolar capillary bed. The bronchial circulation supplies nutrition to the large bronchi and blood vessels, the pleura, and much of the interstitium associated with these structures ${ }^{4}$. In the current four cases, accumulation of hemosiderin-laden macrophages were observed around small- to medium-sized vessels as well as larger vessels adjacent to bronchi, suggesting that vessels associated with both pulmonary and bronchial circulation were affected. However, hemosiderin deposition was most frequently observed around small- to medium-sized vessels within interlobular areas and subpleural areas. Thus, it is likely that feeding vessels such as bronchial arteries and veins are more susceptible compared with pulmonary arteries and veins.

Dark brown to black pigmentation observed in alveolar macrophages with a perivascular or peribronchial distribution is occasionally associated with lung mites (Pneumonyssus sp.) in cynomolgus monkeys ${ }^{1,5}$, however, in the current four cases, these mites were not found.

Although brown pigmentation in the lungs of cynomolgus monkeys is a well-known spontaneous finding, to our knowledge, this is the first report providing the detailed features and distribution of brown pigments and the associated vascular lesions. We believe that this report can help to further understand the underlying pathogenesis of this spontaneous lesion found in cynomolgus monkeys used in preclinical toxicity studies. It may be important when differentiating drug-induced lesions from spontaneous findings, particularly in the event of dealing with drugs affecting the vascular system.

Acknowledgments: We would like to thank all members of the pathology group of Astellas Research Technologies Co., Ltd. for their excellent technical assistance.

Disclosure of Potential Conflicts of Interest: The authors declare that they have no conflict of interest.

\section{References}

1. Chamanza R, Marxfeld HA, Blanco AI, Naylor SW, and Bradley AE. Incidences and range of spontaneous findings in control cynomolgus monkeys (Macaca fascicularis) used in toxicity studies. Toxicol Pathol. 38: 642-657. 2010. [Medline] [CrossRef]

2. Sato J, Doi T, Kanno T, Wako Y, Tsuchitani M, and Narama I. Histopathology of incidental findings in cynomolgus monkeys ( macaca fascicularis ) used in toxicity studies. J Toxicol Pathol. 25: 63-101. 2012. [Medline] [CrossRef]

3. Ito T, Chatani F, Sasaki S, Ando T, and Miyajima H. Spontaneous lesions in cynomolgus monkeys used in toxicity studies. Jikken Dobutsu 41: 455-469.1992.

4. Caswell JL, and Williams KJ. Respiratory system. In: Jubb, Kennedy, and Palmer's Pathology of domestic animals, 5th ed. M.Grant Maxie (eds.). Elsevier Ltd, Philadelphia. 525526. 2007.

5. Osborn KG, and Lowenstine LJ. Respiratory Diseases. In: Nonhuman Primates in Biomedical Research Diseases, Bennett BT, Abee CR, and Henrickson R (eds.). Academic Press, California. 302-303.1998. 